\title{
Cardiovascular Toxicity of Immune Checkpoint Inhibitors: Clinical Risk Factors
}

\author{
Flora Pirozzi ${ }^{1}$ - Remo Poto ${ }^{1}$ - Luisa Aran ${ }^{1}$ - Alessandra Cuomo ${ }^{1}$ Maria Rosaria Galdiero ${ }^{1,2,3}$ - Giuseppe Spadaro ${ }^{1,2,3}$. \\ Pasquale Abete ${ }^{1}$. Domenico Bonaduce ${ }^{1}$ - Gianni Marone ${ }^{1,2,3}$. Carlo Gabriele Tocchetti ${ }^{1,2,4}$ (D) $\cdot$ Gilda Varricchi ${ }^{1,2,3}$. \\ Valentina Mercurio ${ }^{1}$
}

Accepted: 17 December 2020 / Published online: 7 January 2021

(C) The Author(s), under exclusive licence to Springer Science+Business Media, LLC part of Springer Nature 2021

\begin{abstract}
Purpose of Review Immune checkpoint inhibitors, such as monoclonal antibodies targeting CTLA-4, PD-1, and PD-L1, have improved the outcome of many malignancies, but serious immune-related cardiovascular adverse events have been observed. Patients' risk factors for these toxicities are currently being investigated.

Recent Findings Interfering with the CTLA- 4 and PD-1 axes can bring to several immune-related adverse events, including cardiotoxic events such as autoimmune myocarditis, pericarditis, and vasculitis, suggesting that these molecules play an important role in preventing autoimmunity.

Summary Risk factors (such as pre-existing cardiovascular conditions, previous and concomitant cardiotoxic treatments, underlying autoimmune diseases, tumor-related factors, simultaneous immune-related toxic effects, and genetic factors) should be always recognized for the correct management of these toxicities.
\end{abstract}

Keywords Immune checkpoint inhibitors $\cdot$ Cardio-immuno-oncology $\cdot$ Cardiotoxicity $\cdot$ Risk factors

\section{Introduction}

Over the past decade, cancer therapy has been revolutionized by the development of immunotherapy [1]. Various types of immunotherapies have been developed; in particular, immune checkpoint inhibitors (ICIs) have dramatically changed the outcome for many groups of patients with advanced cancer

This article is part of the Topical Collection on Immuno-oncology

Carlo Gabriele Tocchetti

carlogabriele.tocchetti@unina.it

1 Department of Translational Medical Sciences, Federico II University, Naples, Italy

2 Center for Basic and Clinical Immunology Research, Federico II University, Naples, Italy

3 World Allergy Organization Center of Excellence, Federico II University, Naples, Italy

4 Interdepartmental Center for Clinical and Translational Research (CIRCET), Federico II University, Naples, Italy
[2]. Indeed, for decades, oncologists have been developing strategies to modulate inflammation in order to achieve therapeutic anticancer immune responses [3, 4]. The first attempts were not really successful since cancer escapes $\mathrm{T}$ cellmediated cancer-specific immunity via inhibitory pathways mediated by cytotoxic $\mathrm{T}$ lymphocyte-associated protein 4 (CTLA-4), programmed cell death protein 1 (PD-1), and programmed cell death ligand 1 (PD-L1) (all depressing the antineoplastic activity of T lymphocytes). On the opposite, in the last years, ICIs, such as monoclonal antibodies (mAbs) targeting CTLA-4, PD-1, and PD-L1, have improved the outcome of many malignancies, but serious immune-related car-

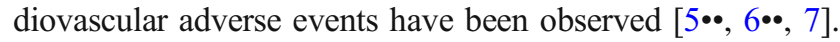
Interfering with the CTLA-4 and PD-1 axes can result in autoimmune myocarditis and dilated cardiomyopathy [8•], suggesting that these molecules play an important role in preventing autoimmunity [9] (Fig. 1). Inhibitors of CTLA-4 include ipilimumab; inhibitors of PD-1 include nivolumab, pembrolizumab, and cemiplimab; and inhibitors of PD-L1 include atezolizumab, avelumab, and durvalumab (https:// www.fda.gov/drugs/resources-information-approved-drugs/ hematologyoncology-cancer-approvals-safety-notifications). These ICIs can also be used in combination (e.g., ipilimumab 
a

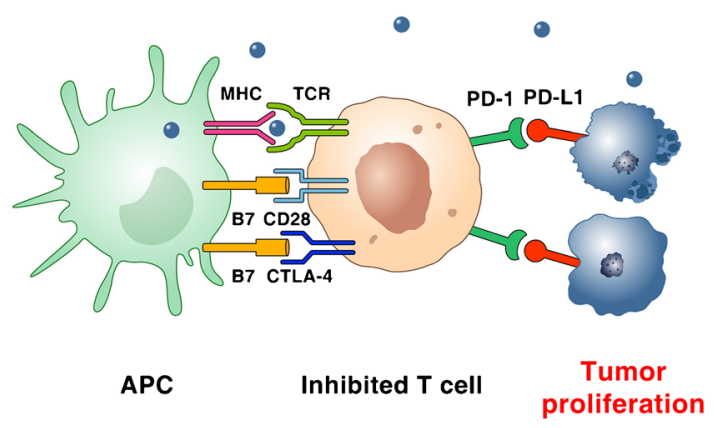

b

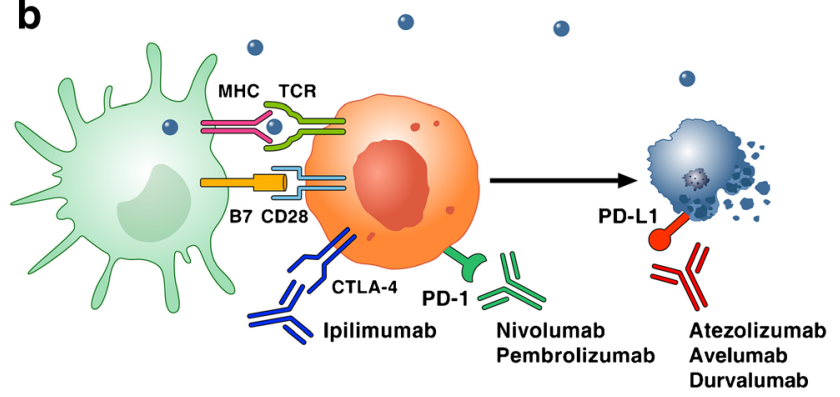

Activated $\mathrm{T}$ cell

C

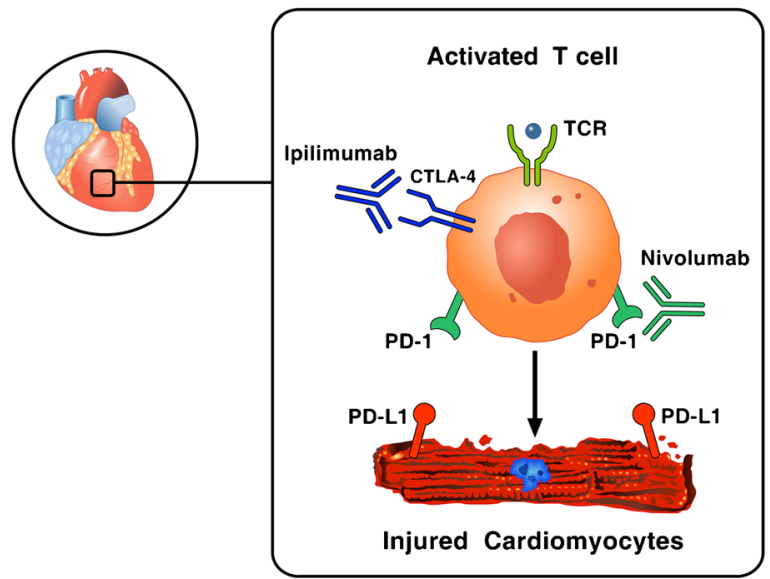

plus nivolumab) in order to increase anticancer activity or can be combined with other cancer therapies [10].

Other immune checkpoints are represented by T-cell immunoglobulin and mucin-containing protein 3 (TIM-3) [11], lymphocyte-activated gene-3 (LAG-3) [12], T-cell immunoreceptor with Ig and ITIM domains (TIGIT) [13], B and T lymphocyte attenuator (BTLA) [14], and V domain Ig suppressor of T-cell activation (VISTA, also known as PD-1 homolog, or PD-1H) [15] and are currently being investigated for their therapeutic potential.

ICIs are now being used for the treatment of advanced metastatic cancers, including melanoma, non-small cell lung cancer (NSCLC), renal cell carcinoma, head and neck squamous cell carcinoma, urothelial cancer, refractory Hodgkin's lymphoma, and malignancies with microsatellite instability

Fig. 1 Reproduced with permission from [9]. a Tumor cells escape immune surveillance by promoting checkpoint activation. Tumor cells express the immune checkpoint activator PD-L1 and produce antigens (blue dots) that are captured by antigen presenting cells (APCs). These cells present antigens to cytotoxic $\mathrm{CD} 8^{+} \mathrm{T}$ cells through the interaction of major histocompatibility complex (MHC) molecules and T-cell receptor (TCR). T-cell activation requires co-stimulatory signals mediated by the interaction between B7 and CD28. Inhibitory signals from CTLA-4 and PD-1 checkpoints dampen T-cell response and promote tumor proliferation. b Checkpoint inhibitors stimulate T-cell activation. Monoclonal antibodies targeting CTLA-4 (ipilimumab), PD-1 (nivolumab, pembrolizumab), and PD-L1 (atezolizumab, avelumab, durvalumab) block immune inhibitory checkpoints (CTLA-4, PD-1, and PD-L1, respectively) and restore anti-tumor immune response, resulting in tumor cell death via release of cytolytic molecules (e.g., TNF- $\alpha$, Granzyme B, IFN- $\gamma$ ). c Hypothetical mechanism by which checkpoint inhibitors can promote autoimmune lymphocytic myocarditis. PD-L1 is expressed in human and murine cardiomyocytes and its expression can increase during myocardial injury. Combination of checkpoint blockade (ipilimumab plus nivolumab) unleashes immune responses and can cause autoimmune lymphocytic myocarditis. Importantly, lymphocytes in myocardium and tumors showed clonality of TCR suggesting that heart and tumors can share antigens (blue dot) recognized by the same $\mathrm{T}$-cell clones

$[5 \cdot \bullet, 16]$ (Table 1). Unfortunately, if on the one hand the activation of the immune system can determine the regression of serious tumors in some patients, on the other hand it can determine the appearance of immune-related adverse events (irAEs) $[17,18]$. These immune-mediated toxicities occur among $70-90 \%$ of patients treated with ICIs [17] and include hepatitis, colitis, thyroiditis, pneumonitis, myositis, dermatitis, and hypophysitis [19]. They are typically reversible and can usually be controlled with glucocorticoid therapy [20]. ICI treatments have been also shown to lead to cardiovascular adverse reactions (CTX); immune-mediated CTX has been observed in individual cases with different presentations $[6 \bullet \bullet]$. In this manuscript, we describe risk factors and clinical manifestations of these cardiotoxicities.

\section{Myocarditis}

The incidence of ICI-associated myocarditis has been estimated to be between 0.27 and $1.14 \%$ in retrospective studies. $[21 \bullet \bullet, 22 \bullet \bullet]$

ICI-associated myocarditis can look like classic myocarditis, and the clinical presentations include signs of acute heart failure (chest pain, shortness of breath, pulmonary edema, and even cardiogenic shock), but can also present with arrhythmias, including heart blocks and both atrial and ventricular arrhythmias that can lead to syncope and sudden death [22••, 23]. In some cases, myocarditis can also be asymptomatic with mild degrees of ventricular dysfunction [24].

Most cases of ICI-associated myocarditis appear early, with a median time of 30 days of toxicity, which corresponds 
Table 1 U.S. FDA-approved immune-checkpoint inhibitors for cancer $[5 \bullet \bullet, 16]$

\begin{tabular}{|c|c|c|}
\hline Checkpoint & Checkpoint inhibitor & Approved uses \\
\hline CTLA-4 & Ipilimumab (Yervoy®) & $\begin{array}{l}\text { Unresectable or metastatic melanoma, alone or combined to ipilimumab, or as adjuvant } \\
\text { Advanced or metastatic renal cell carcinoma, combined to nivolumab } \\
\text { Microsatellite instability-high or mismatch repair-deficient metastatic colorectal cancer alone or combined to } \\
\text { nivolumab } \\
\text { Hepatocellular carcinoma (refractory) in combination with nivolumab } \\
\text { Squamous NSCLC (metastatic) }\end{array}$ \\
\hline \multirow[t]{3}{*}{ PD-1 } & Nivolumab (Opdivo®) & $\begin{array}{l}\text { Unresectable or metastatic melanoma, alone or combined to ipilimumab, or as adjuvant } \\
\text { Metastatic squamous NSCLC } \\
\text { Small cell lung cancer (advanced or metastatic) } \\
\text { Advanced renal cell carcinoma } \\
\text { Relapsed classical Hodgkin's lymphoma } \\
\text { Recurrent or metastatic head-and-neck squamous cell carcinoma } \\
\text { Advanced or metastatic urothelial carcinoma } \\
\text { Microsatellite instability-high or mismatch repair-deficient metastatic colorectal cancer alone or combined to } \\
\text { ipilimumab } \\
\text { Refractory or hepatocellular carcinoma } \\
\text { Advanced or metastatic renal cell carcinoma alone or combined to ipilimumab } \\
\text { Esophageal squamous cell carcinoma (advanced or metastatic) }\end{array}$ \\
\hline & $\begin{array}{r}\text { Pembrolizumab } \\
\left.\text { (Keytruda }{ }^{\circledR}\right)\end{array}$ & $\begin{array}{l}\text { Unresectable or metastatic melanoma } \\
\text { Metastatic NSCLC } \\
\text { Small cell lung cancer (advanced or metastatic) } \\
\text { Recurrent or metastatic head or neck squamous cell carcinoma } \\
\text { Refractory classical Hodgkin's lymphoma } \\
\text { Locally advanced or metastatic urothelial carcinoma } \\
\text { Microsatellite instability-high or mismatch repair-deficient solid tumors and colorectal cancer } \\
\text { Locally advanced or metastatic gastric or gastroesophageal junction adenocarcinoma } \\
\text { Recurrent or metastatic cervical cancer } \\
\text { Refractory primary mediastinal large B-cell lymphoma } \\
\text { Hepatocellular carcinoma } \\
\text { Recurrent or metastatic Merkel cell carcinoma } \\
\text { Renal cell carcinoma (advanced or metastatic) } \\
\text { Cutaneous squamous cell carcinoma (metastatic) }\end{array}$ \\
\hline & Cemiplimab (Libtayo®) & Metastatic cutaneous squamous cell carcinoma \\
\hline \multirow[t]{3}{*}{ PD-L1 } & $\begin{array}{l}\text { Atezolizumab } \\
\quad \text { (Tecentriq }{ }^{\circledR)}\end{array}$ & $\begin{array}{l}\text { Locally advanced or metastatic urothelial carcinoma } \\
\text { Metastatic NSCLC } \\
\text { Extensive-stage SCLC (ES-SCLC) } \\
\text { Triple-negative breast cancer (TNBC) } \\
\text { Hepatocellular carcinoma (unresectable or metastatic) }\end{array}$ \\
\hline & Avelumab (Bavencio®) & $\begin{array}{l}\text { Metastatic Merkel cell carcinoma } \\
\text { Locally advanced or metastatic urothelial carcinoma }\end{array}$ \\
\hline & Durvalumab (Imfinzi®) & $\begin{array}{l}\text { Locally advanced or metastatic urothelial carcinoma } \\
\text { Unresectable stage III NSCLC } \\
\text { Extensive-stage SCLC (ES-SCLC) }\end{array}$ \\
\hline
\end{tabular}

Checkpoints under investigation and that have not undergone FDA approval

TIM-3, LAG-3, TIGIT, BTLA, VISTA (PD-1H)

CTLA-4, cytotoxic T lymphocyte-associated protein-4; PD-1, programmed cell death protein-1; PD-L1, programmed cell death 1 ligand-1; TIM-3, T-cell immunoglobulin and mucin containing protein 3; LAG-3, lymphocyte-activated gene-3; TIGIT, T-cell immunoreceptor with Ig and ITIM domains; BTLA, B- and T-lymphocyte attenuator; VISTA, also known as PD-1 homolog, or PD-1H, V domain Ig suppressor of T-cell activation. Approved indications are up to date as of July 2020

approximately to the first or second administration of ICIs $[25 \bullet \bullet$.

The seminal paper from Mahmood and collaborators [22••] showed that pre-existing cardiovascular risk factors and disease are common in patients on ICIs (both in cases of myocarditis and in controls without myocarditis) [6••].
Combination ICI therapy (e.g., a CTLA-4 inhibitor combined with a PD-1 inhibitor) is the predominant risk factor for ICIassociated myocarditis [21••]. Moreover, myocarditis due to combination ICI therapy is also more severe than the one related to ICI monotherapy [21••]. There appears to be no correlation between ICI-associated myocarditis and age since 
this complication seems to occur in a broad age range (2090 years) $[7,22 \bullet \bullet, 25 \bullet \bullet, 26]$. Toxicity reports show a possible predominance of this adverse reaction in males, but this does not indicate a predisposition of male compared to female sex, given that males are more represented in both ICI use and clinical trial enrollment [25••]. Other risk factors, theoretically, include other treatments (e.g., use of other cardiotoxic antineoplastic agents such as anthracyclines, anti-ErbB2 drugs, Raf and MEK inhibitors, and VEGF tyrosine kinase inhibitors) [27], underlying autoimmune diseases (e.g., rheumatoid arthritis, lupus erythematosus, sarcoidosis), tumor-related factors (cardiac antigens expressed in tumor, activation of T-cell clones against cardiac antigens), simultaneous immunerelated toxic effects (ICI-related skeletal myositis), and genetic factors (gene polymorphisms of CTLA-4, PD-1, or PDL-1) $[6 \bullet \cdot]$. Recent observations also report a protective role for flu vaccination (FV) in the development of ICIs myocarditis and major adverse cardiac events (MACE). In particular, subjects who presented with ICI myocarditis had lower rates of FV. FV was also associated with a lower rate of ICI-related pneumonitis, and myocarditis cases had lower troponin levels and lower rates of cumulative MACE at follow-up in the FV population [28].

Laboratory tests and instrumental examinations are useful for the diagnosis of myocarditis and may reveal patients at higher risk. All diagnostic tests should be used wisely and guided by clinical reasoning. Markers of myonecrosis, including cardiac troponin (cTn), CK-MB (creatine kinase-muscle/ brain), or total CK (creatine kinase), are useful for the diagnosis of myocarditis. In particular, it has been seen that troponin is the preferred biomarker, and since troponin $\mathrm{T}$ may also be elevated because of myositis, troponin I is preferred for establishing cardiac injury [29]. Interestingly, a discharge cardiac troponin $\mathrm{T}$ of more than $1.5 \mathrm{ng} / \mathrm{mL}$ has been reported to be associated with a fourfold increased risk of a major adverse cardiovascular event [22••]. Natriuretic peptides (B-type natriuretic peptide and NT-proBNP) may be elevated in patients with left ventricular dysfunction and heart failure and are frequently elevated in ICI-associated myocarditis [30]. However, BNP is not very specific because it can be chronically elevated in many cancer patients due to cancer-related inflammation [31] and to cancer progression and severity, suggesting the presence of subclinical functional and morphological heart damage in cancer patients [32].

Patients with conduction disease due to ICI therapy in the absence of generalized myocarditis tend to develop a potentially severe ICI-mediated sudden death. This evidence supports the importance of routine ECG monitoring with associated detailed evaluations (e.g., cardio-oncology referral for clinical assessment, cardiac imaging, and 48-h Holter ECG monitoring) of patients on ICI therapy presenting with palpitations, presyncope, or syncope. The ECG may show several changes, including intraventricular conduction delay, PR interval prolongation, various forms of arrhythmias, and eventually complete heart block [21••, 7], Therefore, ECG is neither sensitive nor specific enough for myocarditis screening.

Traditional echocardiography is also not specific for myocarditis and loses sensitivity in case of preserved systolic function $[33,34]$. On the opposite, a recent study compared echocardiographic global longitudinal strain (GLS) in patients receiving ICI who had myocarditis and in patients receiving ICI without myocarditis. The authors showed that GLS decreases in patients with ICI myocarditis and is lower than in patients with ICI without myocarditis. Interestingly, GLS is lower in patients with ICI myocarditis presenting with both a preserved and reduced ejection fraction (EF). Importantly, the study also showed that, during follow-up, patients presenting with low GLS have a higher risk for major adverse cardiac events (MACE), including cardiovascular death, cardiogenic shock, cardiac arrest, and hemodynamically significant complete heart block [35॰].

Cardiac magnetic resonance imaging (CMR) is the preferred instrumental examination for the diagnosis of myocarditis [36]. In a recently published study, Zhang and coworkers evaluated CMR with the use of advanced tissue characterization techniques such as late gadolinium enhancement (LGE) and highlighted that the presence of LGE was modestly associated with histopathological findings and not associated with MACE. These data suggest caution in using a single method such as LGE/standard T2 imaging for the diagnosis of ICI-associated myocarditis, especially in patients with preserved EF [37]. Furthermore, for diagnosis of myocarditis, reviewed Lake Louise Criteria suggest a combination of T2weighted images or T2 mapping and at least one additional T1-based tissue characterization technique [38].

The gold standard for diagnosis of myocarditis is histopathological evidence on endomyocardial biopsy or autopsy [39]. Recently, Champion and Stone described two forms of ICI myocarditis, a high-grade form, in which patients with an increased inflammatory cell infiltration $\left(>50 \mathrm{CD}^{+}\right.$cells/ HPF) had a higher risk of fulminant clinical course, and a low-grade form $\left(<50 \mathrm{CD}^{+}\right.$cells/HPF $)$associated with a better clinical course [40].

Treatment for patients with ICI-associated myocarditis consists in cessation of ICI therapy and immunosuppression with high-dose corticosteroids ( $1-2 \mathrm{mg} / \mathrm{kg} /$ day), until recovery of cardiac function and identification of the triggers. However, discontinuation of immunotherapy should be evaluated individually in case of possible myocarditis. Treatment should be started promptly, given the risk of rapid progression to fulminant disease with cardiac impairment. [41] Recent data suggested that rapid initiation of high doses of corticosteroids is associated with recovery of left ventricular function and can reduce the number of MACE, with patients treated with low doses of corticosteroids being at higher risk of severe outcome $[7,22 \bullet, 42 \bullet]$. In case of therapeutic failure with 
corticosteroids, the use of mycophenolate mofetil, methotrexate, calcineurin inhibitors, intravenous immunoglobulin (IVIG), antithymocyte globulin, rituximab, or infliximab is indicated. [6, 43] However, it should be noted that infliximab may be associated with worsening heart failure and is therefore not indicated in patients with moderate-to-severe heart failure.

A case of severe ICI-related myocarditis unresponsive to high-dose corticosteroid therapy treated with abatacept (CTLA-4 agonist) has been reported [44]. In addition, alemtuzumab, a humanized anti-CD52 monoclonal antibody, has shown significant resolution of cardiotoxicity in a steroidrefractory autoimmune myocarditis induced by PD-1 therapy [45]. Recently, tocilizumab, an IL-6 receptor antagonist, also showed successful improvement of symptoms in a severe ICIrelated myocarditis [46].

\section{Pericardial Disease}

The use of ICIs can also bring to pericardial diseases [25••]. Clinical presentation may include pericarditis, [47, 48] pericardial effusion $[47,49,50]$, or cardiac tamponade [48, 49, 51]. Pericarditis may manifest as chest pain, a new pericardial effusion on the echocardiogram, PR depression and widespread ST elevation on the ECG, or ongoing pericardial inflammation on cMRI or cardiac PET/CT [52].

Treatment for this condition is ICI withdrawal and administration of immunosuppressive therapy with corticosteroids. Colchicine and non-steroidal anti-inflammatory drugs (NSAIDs) are also used for cardiac support as in non-ICIassociated pericarditis [52]. Pericarditis and pericardial effusion can lead to cardiac tamponade, which is a complication that can be fatal. In this case, emergency pericardiocentesis and hemodynamic support appear useful [52].

Salem and colleagues observed that patients receiving antiPD1 or anti-PDL1 therapy for lung cancer had a higher risk of pericardial diseases [25••]. The correlation between this manifestation and lung cancer is unclear. Radiotherapy and immunotherapies might synergize in patients with lung cancer. Pericardial diseases occur more frequently in patients receiving anti-PD-1 or anti-PDL-1 antibodies than those treated with anti-CTLA4. Instead, there was no difference between patients treated with monotherapy and those treated with combination therapy [25••]. The authors hypothesize that cancer patients receiving ICI after thoracic radiotherapy might be more prone to developing pericardial diseases due to the exposure of potentially shared antigens to T-cell recognition. However, the over-reported pericardial diseases might also be explained by the fact that serous illnesses are common cancer complications [53].

As for myocarditis, age does not seem to be a risk factor for ICI-associated pericardial diseases [25]. On the other hand, gender seems to play a role in the risk for these manifestations since they appear to be more common in males than females, as demonstrated by previous studies." Nevertheless, this may simply reflect the demographics of ICI use and clinical trial enrolment [54]. Indeed, a higher number of male patients has been enrolled in clinical trials, with women being excluded for higher incidence of autoimmune diseases and lower risk of cardiovascular diseases. In fact, the true incidence of cardiac toxicity in women is underestimated and the effect of gender on irAEs has not been clearly defined. The apparent sex differences on cardiac toxicity during ICI remains controversial, and therefore further specific studies on this association are needed.

\section{Vasculitis}

Vasculitis is another important MACE. It may affect blood vessels of various sizes, and is typically classified into large, medium, and small-vessel vasculitis based on the size of the vessels involved [55]. In their pharmacovigilance study, Salem and co-workers observed vasculitis disorders associated with ICIs, particularly temporal arteritis and polymyalgia rheumatica [25••]. This inflammatory condition results in end-organ damage. Temporal arteritis is an autoimmune and inflammatory pathology of the aorta and its branches. It presents with headache, jaw claudication, amaurosis fugax or diplopia, and systemic symptoms such as fatigue, fever, and weight loss. Obviously, this condition is associated with an increase in inflammatory indices such as erythrocyte sedimentation rate and C-reactive protein [56]. The gold standard for diagnosis is temporal artery biopsy, which would demonstrate $\mathrm{CD}^{+}{ }^{+}$cells and macrophages organized in granulomas. Color-Doppler ultrasonography of the temporal artery is a non-invasive alternative but is not decisive. Median time to onset of toxicity is 55 days, which corresponds more or less to three ICI administration. As in myocarditis, ICI-mediated toxicity is more frequent in males than in females, and there is no age predisposition [25••]. In addition to stopping treatment, given the risk of vision loss, immunosuppressive treatment should be started early.

Large vessel vasculitis including GCA and aortitis as well as primary angiitis of the nervous system are the most common types of vasculitis associated with immune checkpoint blockade, in particular, anti-PD-1 (e.g., nivolumab) [56]. This is corroborated by the fact that single-nucleotide polymorphisms in the genes encoding PD-1 have been associated with T-cell hyperactivity at a vascular level [57]. Furthermore, Sakthivel and co-workers have shown that polymorphisms of the CTLA-4 gene, which is in close proximity to the PD1 gene on chromosome 2, is associated with granulomatosis with polyangiitis (Wegener's; GPA) [58]. This detection was confirmed in two other studies that showed an association between CTLA-4 gene polymorphisms and GPA [59, 57],

Although the complete pathogenesis of vasculitis has not yet been elucidated, genome-wide association studies have 
underlined the relationship between vasculitis and several genetic polymorphisms.

Importantly, temporal arteritis is more frequent in patients who received anti-CTLA-4 monotherapy than in those who received anti-PD1 or anti-PD-L1 monotherapy. This difference is not detectable in case of vasculitis or polymyalgia rheumatica ICI-related. However, there is no difference between patients treated with combination therapy versus monotherapy. Therefore, vasculitis can be associated with ICI therapy but at the same time they can be a paraneoplastic manifestation of many cancers, first of all melanoma [60, 61]. In particular, temporal arteritis was over-reported for patients with melanoma treated with ipilimumab. Likely, the use of ICIs could reproduce an immune micro-environment favorable for the development of vasculitis, with possible complications such as temporal arteritis and blindness [25].

\section{Covid-19 and the Risk of Cardiotoxicity}

The coronavirus disease 2019 (Covid-19) can particularly affect people with cardiovascular disease through a hyperinflammatory state with resulting cardiotoxicity. Indeed, early data suggest hyper-inflammation and depletion of $\mathrm{T}$ cells with increased expression of PD-1 and PD-L1 during SARS-CoV-2 infection. For this reason, blockade of these crucial pathways with ICIs might be dangerous. Moreover, promoting the immune system with a CTLA-4 agonist may be useful [62]. However, stopping ICI therapy during SARS-CoV-2 infection should be guided in a multidisciplinary approach, and the long-term effects of Covid-19induced hyper-inflammation to the cardiovascular system need further investigations.

\section{Conclusions}

ICIs are associated with cardiovascular toxicities such as myocarditis, pericardial disease, and vasculitis. To date, only a few risk factors have been correlated with the development of ICImediated cardiotoxicity [6••] (Table 2). Hence, a multidisciplinary approach involving cardiologists, oncologists, and immunologists is needed for management of cardiovascular toxicities. Finally, since patients have different risks of developing ICI cardiotoxicity, a personalized clinical approach should be crucial.

The risk factor with the foremost evidence is the combination of two ICIs or concomitant treatment with other cardiotoxic drugs. An in-depth baseline assessment should be performed for all patients, and surveillance strategies on high-at risk patients should be considered for rapidly detecting irAEs, focusing on the first 12 weeks (four cycles), when the risk of developing cardiotoxicity seems to be higher. It has not been clarified whether history of previous myocardial damage
Table 2 Potential risk factors for immune checkpoint inhibitor cardiotoxicity $[6,56-58,35,42]$

Pharmacological history

-Previous treatment with other cardiotoxic therapies (i.e., anthracyclines, anti-ErbB2 drugs, proteasome inhibitors, Raf and MEK inhibitors,

VEGF tyrosine kinase inhibitors, CAR T-cell therapy)

Treatment-related factors

-Combination of two immune checkpoint inhibitors

- Concomitant treatment with other cardiotoxic therapies

-Radiotherapy-induced heart disease

Autoimmune diseases

-Rheumatoid arthritis

-Systemic lupus erythematosus

-Sarcoidosis

-Dressler's syndrome

Cardiovascular factors

-Hypertension

- Coronary artery disease

- Heart failure

-Myocardial infarction

-Myocarditis

-Diabetes mellitus

-Dyslipidemia

- $\uparrow$ cTn; $\downarrow$ GLS; ECG conduction abnormalities

Tumor-related factors

- Cardiac antigens expressed in tumor

-Activation of T-cell clones against cardiac antigens

Genetic factors

-Gene polymorphisms of CTLA-4, PD-1, or PDL-1

(e.g., myocardial infarction or myocarditis) with exposure of cardiac antigens to the immune system is a risk factor for ICImediated cardiotoxicity. Patients with a known history of autoimmune disorders - including rheumatoid arthritis, systemic lupus erythematosus, and sarcoidosis - might be at a higher risk of autoimmune disease, especially with a previous cardiac involvement. Moreover, patients with ICI-related skeletal myositis should perform a cardiological evaluation (ECG, serum natriuretic peptides, cTn measurements, echocardiography) to exclude cardiac involvement. This cardiac assessment may also help to identify ICI-mediated myocarditis in patients presenting with ICI-related skeletal myositis.

The study of genetic factors such as gene polymorphisms of immune checkpoints, and of tumor-related factors as the expression of cardiac antigens in tumor samples, or the activation of T-cell clones against exposed cardiac antigens are intriguing, but they cannot be considered routinely available in real life (Table 2).

These immune-mediated adverse effects require even discontinuation of immunotherapy and personalized immunosuppressive treatment for the risk of fatal adverse events [63]. Since many patients are already on ICI therapy, and considering that the use of ICIs is expected to increase in the coming years, [64] more studies are needed to better understand ICI toxicities and their related risk factors and therefore also to evaluate new therapeutic perspectives. 


\section{Compliance with Ethical Standards}

Conflict of Interest Flora Pirozzi, Remo Poto, Luisa Aran, Alessandra Cuomo, Giuseppe Spadaro, Pasquale Abete, Domenico Bonaduce, and Gilda Varricchi declare no conflict of interest. Maria Rosaria Galdiero is supported, in part, by a grant from the Italian Ministry of Education, University and Research (MIUR). Gianni Marone has received research funding through grants from the Regione Campania CISI-Lab, CRÈME Project, TIMING Project, and Federico II University. Carlo G. Tocchetti has received compensation from Alere for service as a consultant; is listed as an inventor on and receives royalties for Canadian Patent No. 2,613,477 (Thiol-Sensitive Positive Inotropes), issued 3 December 2013, owned by Johns Hopkins University (JHU Reference: C04755P04755-05); and is listed as an inventor on U.S. Patent No. US20180271931 (P75NTR Antagonists and Treatment of Acute and Chronic Cardiac Disease), issued in 2018. Valentina Mercurio has received research funding from MSD.

Human and Animal Rights and Informed Consent This article does not contain any studies with human or animal subjects performed by any of the authors.

\section{References}

Papers of particular interest, published recently, have been highlighted as:

- Of importance

•. Of major importance

1. Farkona S, Diamandis EP, Blasutig IM. Cancer immunotherapy: the beginning of the end of cancer? BMC Med. 2016;14:73.

2. Sharma P, Allison JP. Immune checkpoint targeting in cancer therapy: toward combination strategies with curative potential. Cell. 2015;161:205-14.

3. Lesterhuis WJ, Haanen JB, Punt CJ. Cancer immunotherapyrevisited. Nat Rev Drug Discov. 2011;10:591-600.

4. Tocchetti CG, Ameri P, de Boer RA, D'Alessandra Y, Russo M, Sorriento D, et al. Cardiac dysfunction in cancer patients: beyond direct cardiomyocyte damage of anticancer drugs. Novel cardiooncology insights from the joint 2019 meeting of the ESC working groups of myocardial function and cellular biology of the heart. Cardiovasc Res. 2020;116(11):1820-1834. https://doi.org/10. 1093/cvr/cvaa222.

5.• Hu JR, Florido R, Lipson EJ, Naidoo J, Ardehali R, Tocchetti CG, et al. Cardiovascular toxicities associated with immune checkpoint inhibitors. Cardiovasc Res. 2019;115:854-68 State-of-the-art paper on the cardiovascular science science behind immunotherapy toxicsities.

6.• Lyon AR, Yousaf N, Battisti NML, Moslehi J, Larkin J. Immune checkpoint inhibitors and cardiovascular toxicity. Lancet Oncol. 2018;19:e447-58 State-of-the-art paper on ICIs and their toxicities.

7. Escudier M, Cautela J, Malissen N, Ancedy Y, Orabona M, Pinto J, et al. Clinical features, management, and outcomes of immune checkpoint inhibitor-related cardiotoxicity. Circulation. 2017;136: 2085-7.

8. Nishimura H, Okazaki T, Tanaka Y, Nakatani K, Hara M, Matsumori A, et al. Autoimmune dilated cardiomyopathy in pd-1 receptor-deficient mice. Science. 2001;291:319-22 Early evidence of autoimmune dilated cardiomyopathy with PD1 deletion.
9. Varricchi G, Galdiero MR, Tocchetti CG. Cardiac toxicity of immune checkpoint inhibitors: cardio-oncology meets immunology. Circulation. 2017;136:1989-92.

10. Larkin J, Chiarion-Sileni V, Gonzalez R, Grob JJ, Cowey CL, Lao $\mathrm{CD}$, et al. Combined nivolumab and ipilimumab or monotherapy in untreated melanoma. N Engl J Med. 2015;373:23-34.

11. Yan J, Zhang Y, Zhang JP, Liang J, Li L, Zheng L. Tim-3 expression defines regulatory $t$ cells in human tumors. PLoS One. 2013;8: e58006.

12. Huang CT, Workman CJ, Flies D, Pan X, Marson AL, Zhou G, et al. Role of lag-3 in regulatory t cells. Immunity. 2004;21:503-13.

13. Yu X, Harden K, Gonzalez LC, Francesco M, Chiang E, Irving B, et al. The surface protein TIGIT suppresses $\mathrm{T}$ cell activation by promoting the generation of mature immunoregulatory dendritic cells. Nat Immunol. 2009;10:48-57.

14. Watanabe N, Gavrieli M, Sedy JR, Yang J, Fallarino F, Loftin SK, et al. BTLA is a lymphocyte inhibitory receptor with similarities to CTLA-4 and PD-1. Nat Immunol. 2003;4:670-9.

15. Lines JL, Pantazi E, Mak J, Sempere LF, Wang L, O'Connell S, et al. Vista is an immune checkpoint molecule for human T cells. Cancer Res. 2014;74:1924-32.

16. Ribas A, Wolchok JD. Cancer immunotherapy using checkpoint blockade. Science. 2018;359:1350-5.

17. Postow MA, Sidlow R, Hellmann MD. Immune-related adverse events associated with immune checkpoint blockade. N Engl J Med. 2018;378:158-68.

18. Brunot A, Grob JJ, Jeudy G, Grange F, Guillot B, Kramkimel N, et al. Association of anti-programmed cell death 1 antibody treatment with risk of recurrence of toxic effects after immune-related adverse events of ipilimumab in patients with metastatic melanoma. JAMA Dermatol. 2020;156:982-6.

19. Johnson DB, Chandra S, Sosman JA. Immune checkpoint inhibitor toxicity in 2018. Jama. 2018;320:1702-3.

20. Friedman CF, Proverbs-Singh TA, Postow MA. Treatment of the immune-related adverse effects of immune checkpoint inhibitors: a review. JAMA Oncol. 2016;2:1346-53.

21.• Johnson DB, Balko JM, Compton ML, Chalkias S, Gorham J, Xu $\mathrm{Y}$, et al. Fulminant myocarditis with combination immune checkpoint blockade. $N$ Engl J Med. 2016;375:1749-55 One of the first evidence of ICI myocarditis.

22.• Mahmood SS, Fradley MG, Cohen JV, Nohria A, Reynolds KL, Heinzerling LM, et al. Myocarditis in patients treated with immune checkpoint inhibitors. J Am Coll Cardiol. 2018;71:1755-64 The largest number of patients with ICI myocarditis.

23. Bonaca MP, Olenchock BA, Salem JE, Wiviott SD, Ederhy S, Cohen A, et al. Myocarditis in the setting of cancer therapeutics: proposed case definitions for emerging clinical syndromes in cardio-oncology. Circulation. 2019;140:80-91.

24. Norwood TG, Westbrook BC, Johnson DB, Litovsky SH, Terry $\mathrm{NL}, \mathrm{McKee} \mathrm{SB}$, et al. Smoldering myocarditis following immune checkpoint blockade. J Immunother Cancer. 2017;5:91.

25.• Salem JE, Manouchehri A, Moey M, Lebrun-Vignes B, Bastarache L, Pariente A, et al. Cardiovascular toxicities associated with immune checkpoint inhibitors: an observational, retrospective, pharmacovigilance study. Lancet Oncol. 2018;19:1579-89 Important characterization of ICI cardiotoxicities.

26. Heinzerling L, Ott PA, Hodi FS, Husain AN, Tajmir-Riahi A, Tawbi $\mathrm{H}$, et al. Cardiotoxicity associated with CTLA4 and PD1 blocking immunotherapy. J Immunother Cancer. 2016;4:50.

27. Tocchetti CG, Gallucci G, Coppola C, Piscopo G, Cipresso C, Maurea $\mathrm{C}$, et al. The emerging issue of cardiac dysfunction induced by antineoplastic angiogenesis inhibitors. Eur J Heart Fail. 2013;15(5):482-489. https://doi.org/10.1093/eurjhf/hft008.

28. Awadalla M, DLA G, Mahmood SS, Alvi RM, Mercaldo ND, Hassan MZO, et al. Influenza vaccination and myocarditis among 
patients receiving immune checkpoint inhibitors. $J$ Immunother Cancer. 2019;7:53.

29. Hughes M, Lilleker JB, Herrick AL, Chinoy H. Cardiac troponin testing in idiopathic inflammatory myopathies and systemic sclerosis-spectrum disorders: biomarkers to distinguish between primary cardiac involvement and low-grade skeletal muscle disease activity. Ann Rheum Dis. 2015;74:795-8.

30. Caforio ALP, Adler Y, Agostini C, Allanore Y, Anastasakis A, Arad M, et al. Diagnosis and management of myocardial involvement in systemic immune-mediated diseases: a position statement of the European Society of Cardiology Working Group on Myocardial and Pericardial Disease. Eur Heart J. 2017;38:264962.

31. Bando S, Soeki T, Matsuura T, Tobiume T, Ise T, Kusunose K, et al. Plasma brain natriuretic peptide levels are elevated in patients with cancer. PLoS One. 2017;12:e0178607.

32. Pavo N, Raderer M, Hulsmann M, Neuhold S, Adlbrecht C, Strunk $\mathrm{G}$, et al. Cardiovascular biomarkers in patients with cancer and their association with all-cause mortality. Heart. 2015;101:1874-80.

33. Skouri HN, Dec GW, Friedrich MG, Cooper LT. Noninvasive imaging in myocarditis. J Am Coll Cardiol. 2006;48:2085-93.

34. Logstrup BB, Nielsen JM, Kim WY, Poulsen SH. Myocardial oedema in acute myocarditis detected by echocardiographic 2D myocardial deformation analysis. Eur Heart J Cardiovasc Imaging. 2016;17:1018-26.

35. Awadalla M, Mahmood SS, Groarke JD, Hassan MZO, Nohria A, Rokicki A, et al. Global longitudinal strain and cardiac events in patients with immune checkpoint inhibitor-related myocarditis. $J$ Am Coll Cardiol. 2020;75:467-78 Important manuscript on echocardiographic predictors of patients with increased risk of developing serious myocarditis.

36. Abdel-Aty H, Boye P, Zagrosek A, Wassmuth R, Kumar A, Messroghli D, et al. Diagnostic performance of cardiovascular magnetic resonance in patients with suspected acute myocarditis: comparison of different approaches. J Am Coll Cardiol. 2005;45: 1815-22.

37. Zhang L, Awadalla M, Mahmood SS, Nohria A, Hassan MZO, Thuny F, et al. Cardiovascular magnetic resonance in immune checkpoint inhibitor-associated myocarditis. Eur Heart $J$. 2020;41:1733-43.

38. Ferreira VM, Schulz-Menger J, Holmvang G, Kramer CM, Carbone I, Sechtem U, et al. Cardiovascular magnetic resonance in nonischemic myocardial inflammation: expert recommendations. J Am Coll Cardiol. 2018;72:3158-76.

39. Kindermann I, Barth C, Mahfoud F, Ukena C, Lenski M, Yilmaz A, et al. Update on myocarditis. J Am Coll Cardiol. 2012;59:779-92.

40. Champion SN, Stone JR. Immune checkpoint inhibitor associated myocarditis occurs in both high-grade and low-grade forms. Modern Pathol. 2020;33:99-108.

41. Ganatra S, Neilan TG. Immune checkpoint inhibitor-associated myocarditis. Oncologist. 2018;23:879-86.

42. Zhang L, Zlotoff DA, Awadalla M, Mahmood SS, Nohria A, Hassan MZO, et al. Major adverse cardiovascular events and the timing and dose of corticosteroids in immune checkpoint inhibitorassociated myocarditis. Circulation. 2020;141:2031-4 Succesful use of steroids in ICI myocarditis.

43. Brahmer JR, Lacchetti C, Schneider BJ, Atkins MB, Brassil KJ, Caterino JM, et al. National Comprehensive Cancer N. Management of immune-related adverse events in patients treated with immune checkpoint inhibitor therapy: American Society of Clinical Oncology clinical practice guideline. J Clin Oncol. 2018;36:1714-68.

44. Salem JE, Allenbach Y, Vozy A, Brechot N, Johnson DB, Moslehi $\mathrm{JJ}$, et al. Abatacept for severe immune checkpoint inhibitorassociated myocarditis. N Engl J Med. 2019;380:2377-9.
45. Esfahani K, Buhlaiga N, Thebault P, Lapointe R, Johnson NA, Miller WH Jr. Alemtuzumab for immune-related myocarditis due to PD-1 therapy. N Engl J Med. 2019;380:2375-6.

46. Doms J, Prior JO, Peters S, Obeid M. Tocilizumab for refractory severe immune checkpoint inhibitor-associated myocarditis. Ann Oncol. 2020;31:1273-5.

47. Yun S, Vincelette ND, Mansour I, Hariri D, Motamed S. Late onset ipilimumab-induced pericarditis and pericardial effusion: a rare but life threatening complication. Case Rep Oncol Med. 2015;2015:794842.

48. de Almeida DVP, Gomes JR, Haddad FJ, Buzaid AC. Immunemediated pericarditis with pericardial tamponade during nivolumab therapy. J Immunother. 2018;41:329-31.

49. Nesfeder J, Elsensohn AN, Thind M, Lennon J, Domsky S. Pericardial effusion with tamponade physiology induced by nivolumab. Int J Cardiol. 2016;222:613-4.

50. Shaheen S, Mirshahidi H, Nagaraj G, Hsueh CT. Conservative management of nivolumab-induced pericardial effusion: a case report and review of literature. Exp Hematol Oncol. 2018;7:11.

51. Kushnir I, Wolf I. Nivolumab-induced pericardial tamponade: a case report and discussion. Cardiology. 2017;136:49-51.

52. Adler Y, Charron P, Imazio M, Badano L, Baron-Esquivias G, Bogaert J, et al. 2015 ESC guidelines for the diagnosis and management of pericardial diseases: the task force for the diagnosis and management of pericardial diseases of the European Society of Cardiology (ESC) endorsed by: The European assocIation for Cardio-thoracic Surgery (EACTS). Eur Heart J. 2015;36:2921-64.

53. Varricchi G, Galdiero MR, Mercurio V, Bonaduce D, Marone G, Tocchetti CG. Pharmacovigilating cardiotoxicity of immune checkpoint inhibitors. Lancet Oncol. 2018;19:1545-6.

54. Conforti F, Pala L, Bagnardi V, De Pas T, Martinetti M, Viale G, et al. Cancer immunotherapy efficacy and patients' sex: a systematic review and meta-analysis. Lancet Oncol. 2018;19:737-46.

55. Jennette JC, Falk RJ, Bacon PA, Basu N, Cid MC, Ferrario F, et al. 2012 revised international Chapel Hill consensus conference nomenclature of vasculitides. Arthritis Rheum. 2013;65:1-11.

56. Daxini A, Cronin K, Sreih AG. Vasculitis associated with immune checkpoint inhibitors-a systematic review. Clin Rheumatol. 2018;37:2579-84.

57. Kamesh L, Heward JM, Williams JM, Gough SC, Chavele KM, Salama A, et al. CT60 and +49 polymorphisms of CTLA 4 are associated with ANCA-positive small vessel vasculitis. Rheumatology. 2009;48:1502-5.

58. Sakthivel P, Giscombe R, Ramanujam R, Lefvert AK. Polymorphisms in PDCD1 gene are not associated with Wegener's granulomatosis. Rheumatol Int. 2009;29:1247-50.

59. Giscombe R, Wang X, Huang D, Lefvert AK. Coding sequence 1 and promoter single nucleotide polymorphisms in the CTLA-4 gene in Wegener's granulomatosis. J Rheumatol. 2002;29:950-3.

60. Veitch D, Tsai T, Watson S, Joshua F. Paraneoplastic polyarteritis nodosa with cerebral masses: case report and literature review. Int J Rheum Dis. 2014;17:805-9.

61. Okada M, Suzuki K, Hidaka T, Shinohara T, Takada K, Nakajima $\mathrm{M}$, et al. Polyarteritis associated with hypopharyngeal carcinoma. Intern Med. 2002;41:892-5.

62. Siddiqi HK, Neilan TG. Covid-19, immuno-oncology and cardiovascular disease: viewpoint from the intersection. J Cardiovasc Transl Res. 2020;13:347-8.

63. Wang DY, Salem JE, Cohen JV, Chandra S, Menzer C, Ye F, et al. Fatal toxic effects associated with immune checkpoint inhibitors: a systematic review and meta-analysis. JAMA Oncol. 2018;4:1721-8.

64. Webster RM. The immune checkpoint inhibitors: where are we now? Nat Rev Drug Discov. 2014;13:883-4.

Publisher's Note Springer Nature remains neutral with regard to jurisdictional claims in published maps and institutional affiliations. 